\title{
A thermoelastoplastic theory for special Cosserat rods
}

2019, Vol. 24(3) 686-700

(C) The Author(s) 2019

Article reuse guidelines:

sagepub.com/journals-permissions

DOI: $10.1|77 / 108| 286517754 \mid 32$

journals.sagepub.com/home/mms

(SSAGE

\section{Smriti}

Department of Applied Mechanics, Indian Institute of Technology Delhi, Hauz Khas, New Delhi, India

\author{
Ajeet Kumar \\ Department of Applied Mechanics, Indian Institute of Technology Delhi, Hauz Khas, New Delhi, India
}

\author{
Alexander Großmann \\ Chair of Applied Mechanics, University of Erlangen-Nuremberg, Erlangen, Germany
}

\author{
Paul Steinmann \\ Chair of Applied Mechanics, University of Erlangen-Nuremberg, Erlangen, Germany
}

Received 23 October 2017; accepted 27 December 2017

\begin{abstract}
A general framework is presented to model coupled thermoelastoplastic deformations in the theory of special Cosserat rods. The use of the one-dimensional form of the energy balance in conjunction with the one-dimensional entropy balance allows us to obtain an additional equation for the evolution of a temperature-like one-dimensional field variable, together with constitutive relations for this theory. Reduction to the case of thermoelasticity leads us to the well-known nonlinear theory of thermoelasticity for special Cosserat rods. Later on, additive decomposition is used to separate the thermoelastic part of the strain measures of the rod from their plastic counterparts. We then present the most general quadratic form of the Helmholtz energy per unit undeformed length for both hemitropic and transversely isotropic rods. We also propose a prototype yield criterion in terms of forces, moments, and hardening stress resultants, as well as associative flow rules for the evolution of plastic strain measures and hardening variables.
\end{abstract}

\section{Keywords}

Cosserat rod, elastoplasticity, yield function, thermomechanics, constitutive modeling

\section{Introduction}

The theory of rods is used in modeling deformations in slender bodies whose dimension in one direction is much longer than that in the other two. The theory differs from classical beam theories, such as the Euler-Bernoulli and Timoshenko beam theories, primarily because the latter theories are geometrically linear, and can model only small rotations of the beam's cross-section. The theory of rods, however, is nonlinear and geometrically 
exact, allowing large rotations of the cross-section. This turns out to be very useful in modeling self folding and supercoiling of DNA, collagen and other biomolecules [1-4], and in modeling of long carbon nanotubes [5-7], hair [8], plant tendrils [9], cables [10,11], catheters [12], etc. The theory for modeling large rotations in a one-dimensional framework originates in Euler's well-known theory of elastica [13], which is only applicable to planar deformations of slender bodies. The theory of Kirchhoff rods [13] generalized Euler's elastica theory to model three-dimensional deformations of slender bodies. It allows arbitrary bending and twisting of the rod but does not allow extension or shearing of the rod's centerline. The theory is further generalized based on the work of the Cosserat brothers [14], who considered the centerline displacement and the cross-section's rotation as independent variables, allowing extension and shear in the rod too. In addition to extension, shearing, bending, and twisting, the general theory of Cosserat rods [15-17] also allows independent (but limited to cross-sectional shearing and anisotropic cross-sectional stretching) deformation of the cross-section. However, the focus of this paper is on a special case of this theory, i.e., the theory of special Cosserat rods [18]. Here, the deformation of the cross-section is assumed to be completely dependent on the local values of extension, shear, bending, and twist. The governing equations of special Cosserat rods can be derived using two approaches: (i) the direct approach [18-20] and (ii) by reduction from the three-dimensional equations of elasticity for slender bodies. The second approach is usually achieved by additional kinematic hypotheses (such as the plane section remains plane), integration of the three-dimensional elasticity equations over the rod's cross-section, series expansion of in-plane and out-of-plane displacements, or through variational asymptotic methods $[6,18,21,22]$. Although the initial applications of the theory were restricted to modeling purely elastic deformations, several variants of the theory have subsequently been proposed to account for thermoelastic [23-26], as well as viscoelastic behavior [27, 28].

The thermoelastic theory of special Cosserat rods has also been derived using both the direct approach $[23-25,29,30]$ and the indirect approach, i.e., through integration of the three-dimensional equations of thermoelasticity in the rod's cross-section [26]. The one-dimensional form of the energy balance in conjunction with the entropy balance is used to obtain the thermoelastic constitutive relations (just the abstract form) as well as the evolution equation for the temperature-like one-dimensional field variable representing the distribution of temperature in the rod's cross-section. Simmonds [26] and Altenbach et al. [29] further refined the theory and introduced two temperature field variables: the first representing the average absolute temperature in the rod's cross-section and the second representing the deviation of temperature within the cross-section. However, the method to obtain the parameters present, even in the linear form of the one-dimensional thermoelastic constitutive relations, from the three-dimensional theory is still missing for general three-dimensional thermoelastic constitutive models. Deriving the nonlinear form of the one-dimensional thermoelastic constitutive relations is an even bigger challenge.

There is a volume of literature on three-dimensional elastoplastic modeling and corresponding modeling in dimensionally reduced structures, such as beams, plates, and shells. Simo and Kennedy [31] proposed a framework for modeling elastoplastic deformations within their two-dimensional shell theory. There are several works accounting for elastoplastic deformations within classical linear beam theories [32, 33]. Simo et al. [34] have pursued the same for planar deformations of their nonlinear beam model. They also proposed a yield function in terms of the axial force, shear force, and bending moment. Park and Lee [35] integrated the three-dimensional elastoplastic constitutive equations within the rod's cross-section to account for nonplanar elastoplastic deformations within the special Cosserat rod theory. However, they assume rigid crosssection kinematics for the integration through the cross-section, which leads to inaccurate constitutive relations even for purely elastic deformations [6]. A direct approach to include elastoplastic deformations within the special Cosserat rod theory is still missing. One challenge is how to propose the kinematics for general threedimensional elastoplastic deformations (choice of plastic strains and hardening variables). Another issue is how to propose the mathematical form of the yield function in terms of the stress resultants. The goal of this paper is to address these challenges and present a direct approach to model thermoelastoplastic deformations within the special Cosserat rod theory.

The outline of the paper is as follows. We begin with a brief overview of the kinematics and dynamics of special Cosserat rods in Section 2. We then discuss the thermodynamics of special Cosserat rods in Section 3. Here, we first present the one-dimensional form of the energy balance and entropy balance specific to special Cosserat rods. We further use the Coleman-Noll exploitation to obtain the functional dependence of constitutive relations. Finally, we obtain the evolution equation of the temperature-like one-dimensional field variable (representing the distribution of absolute temperature in the rod's cross-section). We have kept the treatment general in this section by the inclusion of a general internal variable vector so that the analysis could be extended in 
future, even for viscoelastic or damage modeling in the special Cosserat rod theory. We then specialize to the case of thermoelastoplastic deformations in Section 4. Here, we begin with the kinematics and assume that the total strain measures of the special Cosserat rod additively decompose into elastic and plastic parts. We also define the internal variable vector as comprising the plastic strain measures and the hardening variable. We then assume the rod's Helmholtz energy per unit undeformed length to comprise the thermoelastic stored energy and the hardening energy. The thermoelastic stored energy is further assumed to depend only on the elastic part of the strain, while the hardening energy is assumed to depend on just the hardening variable. We then present the most general quadratic form of the thermoelastic stored energy for hemitropic rods. Finally, we present a discussion on the yield function, the associative flow rule, and the hardening law. Section 5 concludes our paper and proposes several directions for future research.

\section{I.I. Notation}

Unless specified otherwise, lowercase bold letters denote vectors, whereas uppercase bold letters denote secondand higher-order tensors. The corresponding letters when subscripted with zero, "( $)_{0}$ ", denote the (rotational) pull-backs of these vectors and tensors through the rod's cross-sectional rotation. The derivative with respect to time is denoted by an overdot "( $\left.{ }^{\circ}\right)$ ", whereas the derivative with respect to the rod's undeformed arc-length is denoted by a prime " ()$^{\prime \prime}$ ". Finally, repeated Latin indices sum from 1 to 3, whereas repeated Greek indices sum from 1 to 2 .

\section{Brief overview of kinematics and dynamics of special Cosserat rods}

In this section, we present briefly the kinematics and dynamics of special Cosserat rods. ${ }^{1}$

\section{I. Kinematics}

Figure 1 shows a typical rod deforming from its reference configuration, i.e., its straight state. Here $\mathbf{X}=X_{i} \mathbf{e}_{i}$ (with $X_{3}=s$ ), denotes the position vector of a typical material point of a rod in its reference configuration. The components $\left(X_{1}, X_{2}\right)$ denote the coordinates in the rod's undeformed cross-section $(\Omega)$ and $s$ denotes the coordinate along the undeformed arc-length. The variable $\mathbf{r}(s)$ denotes the centerline of the deformed rod. We also have two directors $\left(\mathbf{d}_{1}(s), \mathbf{d}_{2}(s)\right.$ ), which are the images of two orthonormal basis vectors $\left(\mathbf{e}_{1}, \mathbf{e}_{2}\right)$ in the undeformed cross-section of the rod (typically, the material lines along the two principal axes). The third director, $\mathbf{d}_{3}(s)$, is assumed to be perpendicular to the other two. The directors rigidly rotate in this theory and model the orientation of the rod's cross-section, i.e., $\mathbf{d}_{i}(s)=\mathbf{R}(s) \mathbf{e}_{i}$. Here, the quantity $\mathbf{R}(s)$ denotes a threedimensional rotation tensor. The field quantities $(\mathbf{r}, \mathbf{R})$ are thus the kinematic variables of this theory. We further introduce the following quantities and their rotational pull-backs: ${ }^{2}$

$$
\begin{aligned}
& \mathbf{v}=\mathbf{r}^{\prime}=\mathrm{v}_{i} \mathbf{d}_{i}, \quad \mathbf{v}_{0}=\mathbf{R}^{\mathrm{T}} \mathbf{v}=\mathrm{v}_{i} \mathbf{e}_{i} \\
& \mathbf{K}=\mathbf{R}^{\prime} \mathbf{R}^{\mathrm{T}}, \quad \mathbf{k}=\operatorname{axial}(\mathbf{K})=k_{i} \mathbf{d}_{i}, \quad \mathbf{k}_{0}=\mathbf{R}^{\mathrm{T}} \mathbf{k}=k_{i} \mathbf{e}_{i} .
\end{aligned}
$$

The quantities $(\mathbf{v}, \mathbf{k})$ can be thought of as the spatial strain measures in this theory, whereas their rotational pull-backs $\left(\mathbf{v}_{0}, \mathbf{k}_{0}\right)$ can be thought of as the material strain measures. The first two components of $\mathbf{v}_{0}$ represent shear, while the third component represents axial stretch. Similarly, the first two components of $\mathbf{k}_{0}$ represent the components of local bending curvature, while the third component represents twist.

\subsection{Dynamics}

We then let $\mathbf{n}(s)$ and $\mathbf{m}(s)$ denote the internal contact force and the internal moment, respectively, that act on a typical cross-section of a rod and write

$$
\mathbf{n}=n_{i} \mathbf{d}_{i}, \quad \mathbf{m}=m_{i} \mathbf{d}_{i} .
$$

Here, $\left(n_{1}, n_{2}\right)$ are the shear forces, $n_{3}$ is the axial force, $\left(m_{1}, m_{2}\right)$ are the bending moments, and $m_{3}$ is the torque or twisting moment. Their rotational pull-backs are given by

$$
\mathbf{n}_{0}=n_{i} \mathbf{e}_{i}, \quad \mathbf{m}_{0}=m_{i} \mathbf{e}_{i} .
$$




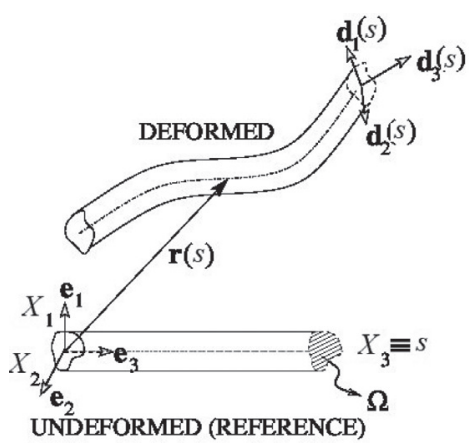

Figure I. A typical rod deforming from its straight state reference configuration.

On linear and angular momentum balance of an arbitrary segment of the rod, one obtains the following equations for the dynamics of the rod:

$$
\mathbf{n}^{\prime}+\hat{\mathbf{n}}=\rho_{0} A_{0} \mathbf{a}, \quad \mathbf{m}^{\prime}+\mathbf{v} \times \mathbf{n}+\hat{\mathbf{m}}=\dot{\overline{\mathbf{I}} \boldsymbol{\omega}}=\mathbf{I} \boldsymbol{\alpha}+\boldsymbol{\omega} \times[\mathbf{I} \boldsymbol{\omega}] .
$$

Here, $\hat{\mathbf{n}}$ and $\hat{\mathbf{m}}$ are the distributed force and the distributed couple, respectively, that act on a rod, whereas $\rho_{0}$ and $A_{0}$ denote the mass per unit undeformed volume and the undeformed cross-sectional area, respectively, of the rod. Furthermore, $\mathbf{a}=\ddot{\mathbf{r}}$ denotes the acceleration of the mass center of the rod's cross-section, whereas $v=\dot{\mathbf{r}}$ denotes its velocity. ${ }^{3}$ The quantity $\omega$ in equation (4b) denotes the angular velocity of the rod's cross-section, which is defined as

$$
\boldsymbol{\omega}=\operatorname{axial}\left(\dot{\mathbf{R}} \mathbf{R}^{\mathrm{T}}\right), \quad \boldsymbol{\omega}_{0}=\mathbf{R}^{\mathrm{T}} \boldsymbol{\omega}=\operatorname{axial}\left(\mathbf{R}^{\mathrm{T}} \dot{\mathbf{R}}\right)
$$

Similarly, $\boldsymbol{\alpha}$ denotes the angular acceleration and is defined as

$$
\boldsymbol{\alpha}=\dot{\boldsymbol{\omega}}, \quad \boldsymbol{\alpha}_{0}=\mathbf{R}^{\mathrm{T}} \boldsymbol{\alpha}=\mathbf{R}^{\mathrm{T}} \dot{\dot{\mathbf{R}} \boldsymbol{\omega}_{0}}=\dot{\boldsymbol{\omega}}_{0}
$$

Finally, $\mathbf{I}=\mathbf{R I}_{0} \mathbf{R}^{\mathrm{T}}$ denotes the moment of inertia tensor of the rod's cross-section whose rotational pull-back $\mathbf{I}_{0}$ is defined as

$$
\begin{aligned}
\mathbf{I}_{0} & =\sum_{j=1}^{3} I_{j} \mathbf{e}_{j} \otimes \mathbf{e}_{j} \quad \text { where }, \\
I_{1} & =\int_{\Omega} \rho_{0} X_{2}^{2} \mathrm{~d} X_{1} \mathrm{~d} X_{2}, \quad I_{2}=\int_{\Omega} \rho_{0} X_{1}^{2} \mathrm{~d} X_{1} \mathrm{~d} X_{2}, \quad I_{3}=I_{1}+I_{2} .
\end{aligned}
$$

Here, $\mathbf{I}_{0}$ assumes the above diagonal form, since we have assumed that $\left(\mathbf{e}_{1}, \mathbf{e}_{2}\right)$ are aligned along the crosssection's principal axes of inertia. The dynamic equations in equation (4) can also be written in terms of the pull-back or material quantities, as follows:

$$
\mathbf{n}_{0}^{\prime}+\mathbf{k}_{0} \times \mathbf{n}_{0}+\hat{\mathbf{n}}_{0}=\rho_{0} A_{0} \mathbf{a}_{0}, \quad \mathbf{m}_{0}^{\prime}+\mathbf{k}_{0} \times \mathbf{m}_{0}+\mathbf{v}_{0} \times \mathbf{n}_{0}+\hat{\mathbf{m}}_{0}=\mathbf{I}_{0} \boldsymbol{\alpha}_{0}+\boldsymbol{\omega}_{0} \times\left[\mathbf{I}_{0} \boldsymbol{\omega}_{0}\right] .
$$

After substitution of the constitutive relations in the case of elasticity (see Section 4.2), the system of dynamic equations in equation (4) or equation (8) becomes second order with respect to the rod's undeformed length. One accordingly requires 12 boundary conditions for the solution of these equations.

\section{Thermodynamics of special Cosserat rods}

Having discussed the kinematics and dynamics of special Cosserat rods, we now turn to their thermodynamics. We will discuss in detail the first and second laws of thermodynamics specific to rods. 


\section{I. The first law of thermodynamics: Balance of energy}

The first law of thermodynamics relates the time rate of the internal energy $(\mathcal{U})$ and the kinetic energy $(\mathcal{K})$ of a system to the external mechanical power $\left(\mathcal{P}^{\text {ext }}\right)$ and the thermal power $(\mathcal{Q})$ supplied to the system. Mathematically, we write this as

$$
\dot{\mathcal{U}}+\dot{\mathcal{K}}=\mathcal{P}^{\text {ext }}+\mathcal{Q}
$$

For a rod of length $L$, these quantities can be expressed as

$$
\begin{aligned}
\mathcal{U} & =\int_{0}^{L} u \mathrm{~d} s, \\
\mathcal{K} & =\int_{0}^{L}\left[\frac{1}{2} \rho_{0} A_{0} \boldsymbol{v} \cdot \boldsymbol{v}+\frac{1}{2}[\mathbf{I} \boldsymbol{\omega}] \cdot \boldsymbol{\omega}\right] \mathrm{d} s, \\
\mathcal{P}^{\mathrm{ext}} & =\left.\left[\mathbf{n}^{\mathrm{ext}} \cdot \boldsymbol{v}+\mathbf{m}^{\mathrm{ext}} \cdot \boldsymbol{\omega}\right]\right|_{0} ^{L}+\int_{0}^{L}[\hat{\mathbf{n}} \cdot \boldsymbol{v}+\hat{\mathbf{m}} \cdot \boldsymbol{\omega}] \mathrm{d} s, \\
\mathcal{Q} & =\int_{0}^{L} \hat{\gamma} \mathrm{d} s-\left.q^{\mathrm{ext}}\right|_{0} ^{L}=\int_{0}^{L}\left[\hat{\gamma}-q^{\prime}\right] \mathrm{d} s .
\end{aligned}
$$

Here, $u$ denotes the internal energy per unit undeformed length of the rod, which is obtained by integrating the bulk internal energy density in the rod's cross-section. The quantity $\hat{\gamma}$ denotes the heat source per unit undeformed length of the rod, which is the resultant of the bulk heat source (integrated over the rod's cross-section) and the inflow of heat flux through the rod's lateral surface (integrated along the cross-section's boundary). The term $q$ denotes the scalar heat flux along the cross-section's normal which is obtained by integrating, in the plane of the cross-section, the projection of the heat flux vector along the cross-section normal. Similarly, $q^{\text {ext }}$ denotes heat flux through the two end cross-sections of the rod for which the relevant boundary condition is prescribed. Finally, $\mathbf{n}^{\text {ext }}$ and $\mathbf{m}^{\text {ext }}$ are the external force and moment, respectively, applied at the two end cross-sections, which are usually specified as boundary conditions, provided the corresponding kinematic quantity $\mathbf{r}$ or $\mathbf{R}$ is not specified. On substituting the definitions in equation (10) into equation (9), we obtain: ${ }^{4}$

$$
\begin{aligned}
& \int u \mathrm{u} s+\frac{\mathrm{d}}{\mathrm{d} t} \int\left[\frac{1}{2} \rho_{0} \mathrm{~A}_{0} \boldsymbol{v} \cdot \boldsymbol{v}+\frac{1}{2}[\mathbf{I} \boldsymbol{\omega}] \cdot \boldsymbol{\omega}\right] \mathrm{d} s \\
&= {\left.\left[\mathbf{n}^{\mathrm{ext}} \cdot \boldsymbol{v}+\mathbf{m}^{\mathrm{ext}} \cdot \boldsymbol{\omega}\right]\right|_{0} ^{L}+\int[\hat{\mathbf{n}} \cdot \boldsymbol{v}+\hat{\mathbf{m}} \cdot \boldsymbol{\omega}] \mathrm{d} s+\int\left[\hat{\gamma}-q^{\prime}\right] \mathrm{d} s } \\
& \Rightarrow \int \dot{u} \mathrm{~d} s+\int\left[\rho_{0} A_{0} \mathbf{a} \cdot \boldsymbol{v}+[\dot{\overline{\mathbf{I}}}] \cdot \boldsymbol{\omega}\right] \mathrm{d} s \\
&=\int\left[[\mathbf{n} \cdot \boldsymbol{v}+\mathbf{m} \cdot \boldsymbol{\omega}]^{\prime}+[\hat{\mathbf{n}} \cdot \boldsymbol{v}+\hat{\mathbf{m}} \cdot \boldsymbol{\omega}]\right] \mathrm{d} s+\int\left[\hat{\gamma}-q^{\prime}\right] \mathrm{d} s \\
& \Rightarrow \int \dot{u} \mathrm{~d} s=\int\left[\mathbf{n}^{\prime}+\hat{\mathbf{n}}-\rho_{0} A_{0} \mathbf{a}\right] \cdot \boldsymbol{v} \mathrm{d} s+\int\left[\mathbf{m}^{\prime}+\hat{\mathbf{m}}-\dot{\mathbf{I} \boldsymbol{\omega}}\right] \cdot \boldsymbol{\omega} \mathrm{d} s+\int\left[\hat{\gamma}-q^{\prime}\right] \mathrm{d} s+\int\left[\mathbf{n} \cdot \boldsymbol{v}^{\prime}+\mathbf{m} \cdot \boldsymbol{\omega}^{\prime}\right] \mathrm{d} s \\
& \Rightarrow \int \dot{u} \mathrm{~d} s=-\int[\mathbf{v} \times \mathbf{n}] \cdot \boldsymbol{\omega} \mathrm{d} s+\int\left[\hat{\gamma}-q^{\prime}\right] \mathrm{d} s+\int\left[\mathbf{n} \cdot \boldsymbol{v}^{\prime}+\mathbf{m} \cdot \boldsymbol{\omega}^{\prime}\right] \mathrm{d} s .
\end{aligned}
$$

As this equation holds for any arbitrary segment of the rod, we obtain the following local form for the balance of energy:

$$
\dot{u}=\mathbf{n} \cdot \boldsymbol{v}^{\prime}+\mathbf{m} \cdot \boldsymbol{\omega}^{\prime}-[\mathbf{v} \times \mathbf{n}] \cdot \boldsymbol{\omega}+\hat{\gamma}-q^{\prime} .
$$

The spatial derivatives of the velocities $\left(\boldsymbol{v}^{\prime}, \boldsymbol{\omega}^{\prime}\right)$ can be further written in terms of the strain rates $\left(\dot{\mathbf{v}}_{0}, \dot{\mathbf{k}}_{0}\right)$ as

$$
\begin{aligned}
\boldsymbol{v}^{\prime} & =\dot{\mathbf{v}} \quad \text { (on interchanging the order of derivative) } \\
& =\dot{\mathbf{R} \mathbf{v}_{0}}=\boldsymbol{\omega} \times \mathbf{v}+\mathbf{R} \dot{\mathbf{v}}_{0}, \\
\omega^{\prime} & =\mathbf{R} \dot{\mathbf{k}}_{0} \quad \text { (see appendix for proof), }
\end{aligned}
$$


which when substituted in equation (11) yields

$$
\begin{aligned}
\dot{u} & =\mathbf{n}_{0} \cdot \dot{\mathbf{v}}_{0}+\mathbf{m}_{0} \cdot \dot{\mathbf{k}}_{0}+\hat{\gamma}-q^{\prime} \\
& =: \mathbf{n}_{0} \cdot \boldsymbol{\varepsilon}_{0}+\mathbf{m}_{0} \cdot \varkappa_{0}+\hat{\gamma}-q^{\prime} .
\end{aligned}
$$

We have purposely introduced two new symbols $\left(\boldsymbol{\varepsilon}_{0}=: \dot{\mathbf{v}}_{0}, \varkappa_{0}=: \dot{\mathbf{k}}_{0}\right)$ here for the material strain and curvature rates. They resemble the notation used for strain and curvature in a geometrically linear setting.

\subsection{The second law of thermodynamics: Balance of entropy}

We now discuss the second law of thermodynamics specific to rods in order to obtain their constitutive relations. Let us define the total entropy of the rod as

$$
\mathcal{H}=\int_{0}^{L} \eta \mathrm{d} s
$$

Here, $\eta$ denotes entropy per unit undeformed length of the rod, which can again be obtained by integrating the bulk entropy density in the rod's cross-section. The time rate of change of the rod's entropy is then assumed to be comprised of two parts:

$$
\dot{\mathcal{H}}=\delta^{\text {ext }}+\delta^{\text {prod }}
$$

where

$$
\begin{aligned}
\delta^{\text {ext }} & =\int_{0}^{L} \frac{\hat{\gamma}}{T} \mathrm{~d} s-\left.\frac{q^{\text {ext }}}{T}\right|_{0} ^{L} \quad(\text { external entropy supply rate }) \\
\delta^{\text {prod }} & =\int_{0}^{L} \frac{\mathfrak{D}}{T} \mathrm{~d} s \geq 0 \quad \text { (internal entropy production rate) }
\end{aligned}
$$

Here, $\mathfrak{D} \geq 0$ denotes the dissipation power per unit undeformed length and is always non-negative, whereas $T>0$ denotes the representative (absolute) temperature of the rod's cross-section. On substituting equations (14) and (16) into equation (15), we obtain

$$
\begin{aligned}
\int_{0}^{L} \dot{\eta} \mathrm{d} s & =\int_{0}^{L}\left[\frac{\hat{\gamma}}{T}-\left[\frac{q}{T}\right]^{\prime}\right] \mathrm{d} s+\int_{0}^{L} \frac{\mathfrak{D}}{T} \mathrm{~d} s \\
& =\int_{0}^{L} \frac{1}{T}\left[\hat{\gamma}-q^{\prime}+\frac{q T^{\prime}}{T}+\mathfrak{D}\right] \mathrm{d} s \\
\Rightarrow T \dot{\eta} & =\hat{\gamma}-q^{\prime}+\frac{q T^{\prime}}{T}+\mathfrak{D} .
\end{aligned}
$$

Here, the last equality is obtained through the usual localization argument for an arbitrary section of the rod. On substituting the expression for $\left[\hat{\gamma}-q^{\prime}\right]$ from the energy balance equation (equation (13)), we eventually obtain the following form of the entropy balance for the rod:

$$
T \dot{\eta}=\dot{u}-\mathbf{n}_{0} \cdot \boldsymbol{\varepsilon}_{0}-\mathbf{m}_{0} \cdot \varkappa_{0}+\frac{q T^{\prime}}{T}+\mathfrak{D} .
$$

\subsection{Coleman-Noll exploitation}

For the rod to satisfy the second law of thermodynamics, it is necessary that the rod's constitutive relations are suitably restricted. We thus hypothesize that all the constitutive processes must be consistent with the Clausius-Duhem inequality, i.e., the dissipation inequality. Let us consider a general Helmholtz energy per unit undeformed length $(\Psi)$ for the rod, i.e.,

$$
\Psi=u-\eta T
$$


having the following functional dependence:

$$
\Psi=\hat{\Psi}\left(\mathbf{v}_{0}, \mathbf{k}_{0}, T, T^{\prime}, \boldsymbol{\beta}_{0}\right)
$$

Here, $\boldsymbol{\beta}_{0}$ represents the vector of internal variables (if any) for the $\operatorname{rod}^{5}$ As we shall see later, $\Psi$ allows us to express all the thermodynamic state variables in terms of the cross-section's representative temperature (rather than $u$ or $\eta$, which are difficult to measure) and other kinematic quantities. To exploit the dissipation inequality, we first write the time rate of $u$ as follows:

$$
\begin{aligned}
\dot{u} & =\dot{\Psi}+\dot{\eta} T+\eta \dot{T} & & \text { (using equation (19)) } \\
& =\partial_{\mathbf{v}_{0}} \Psi \cdot \boldsymbol{\varepsilon}_{0}+\partial_{\mathbf{k}_{0}} \Psi \cdot \varkappa_{0}+\partial_{T} \Psi \dot{T}+\partial_{T^{\prime}} \Psi \dot{T}^{\prime}+\partial_{\beta_{0}} \Psi \cdot \dot{\boldsymbol{\beta}}_{0}+\dot{\eta} T+\eta \dot{T} & & \text { (using equation (20)) }
\end{aligned}
$$

We then use equation (18) to express the dissipation term $\mathfrak{D}$ and further substitute equation (21) into the result to obtain

$$
\begin{aligned}
\mathfrak{D} & =\mathbf{n}_{0} \cdot \boldsymbol{\varepsilon}_{0}+\mathbf{m}_{0} \cdot \varkappa_{0}-\frac{q T^{\prime}}{T}+T \dot{\eta}-\dot{u} \geqslant 0 \\
& =\mathbf{n}_{0} \cdot \boldsymbol{\varepsilon}_{0}+\mathbf{m}_{0} \cdot \varkappa_{0}-\frac{q T^{\prime}}{T}-\eta \dot{T}-\partial_{\mathbf{v}_{0}} \Psi \cdot \boldsymbol{\varepsilon}_{0}-\partial_{\mathbf{k}_{0}} \Psi \cdot \varkappa_{0}-\partial_{T} \Psi \dot{T}-\partial_{T^{\prime}} \Psi \dot{T}^{\prime}-\partial_{\boldsymbol{\beta}_{0}} \Psi \cdot \dot{\boldsymbol{\beta}}_{0} \geqslant 0 \\
& =\left[\mathbf{n}_{0}-\partial_{\mathbf{v}_{0}} \Psi\right] \cdot \boldsymbol{\varepsilon}_{0}+\left[\mathbf{m}_{0}-\partial_{\mathbf{k}_{0}} \Psi\right] \cdot \varkappa_{0}-\left[\eta+\partial_{T} \Psi\right] \dot{T}-\partial_{T^{\prime}} \Psi \dot{T}^{\prime}-\frac{q T^{\prime}}{T}-\partial_{\boldsymbol{\beta}_{0}} \Psi \cdot \dot{\boldsymbol{\beta}}_{0} \geqslant 0 .
\end{aligned}
$$

Assuming that $\left(\mathbf{v}_{0}, \mathbf{k}_{0}, T, T^{\prime}\right)$ and their time rates $\left(\varepsilon_{0}, \varkappa_{0}, \dot{T}, \dot{T}^{\prime}\right)$ can take arbitrarily prescribed and independent values at any point of time, the coefficients of $\left(\varepsilon_{0}, \varkappa_{0}, \dot{T}, \dot{T}^{\prime}\right)$ must vanish independently. This gives us the following constitutive relations for the rod:

$$
\begin{aligned}
\partial_{T^{\prime}} \Psi=0 \Rightarrow \Psi & =\hat{\Psi}\left(\mathbf{v}_{0}, \mathbf{k}_{0}, T, \boldsymbol{\beta}_{0}\right) \quad\left(\Psi \text { is independent of } T^{\prime}\right), \\
\mathbf{n}_{0} & =\partial_{\mathbf{v}_{0}} \hat{\Psi}\left(\mathbf{v}_{0}, \mathbf{k}_{0}, T, \boldsymbol{\beta}_{0}\right) \\
\mathbf{m}_{0} & =\partial_{\mathbf{k}_{0}} \hat{\Psi}\left(\mathbf{v}_{0}, \mathbf{k}_{0}, T, \boldsymbol{\beta}_{0}\right) \\
\eta & =-\partial_{T} \hat{\Psi}\left(\mathbf{v}_{0}, \mathbf{k}_{0}, T, \boldsymbol{\beta}_{0}\right) .
\end{aligned}
$$

Finally, the dissipation $\mathfrak{D}$ reduces to

$$
\begin{aligned}
\mathfrak{D} & =-\frac{q T^{\prime}}{T}-\partial_{\boldsymbol{\beta}_{0}} \Psi \cdot \dot{\boldsymbol{\beta}_{0}} \geq 0 \\
& =-\frac{q T^{\prime}}{T}+\mathbf{A}_{0} \cdot \dot{\boldsymbol{\beta}_{0}} \geqslant 0 \quad\left(\mathbf{A}_{0}:=-\partial_{\boldsymbol{\beta}_{0}} \Psi\right) \\
& =\mathfrak{D}^{\text {cond }}+\mathfrak{D}^{\text {loc }} .
\end{aligned}
$$

Here, $\mathbf{A}_{0}$ is the thermodynamic stress resultant conjugate to the internal variable vector $\boldsymbol{\beta}_{0}$ and $\mathfrak{D}^{\text {cond }}=-q T^{\prime} / T$ denotes dissipation due to heat conduction along the rod's arc-length, while $\mathfrak{D}^{\text {loc }}=\mathbf{A}_{0} \cdot \dot{\boldsymbol{\beta}}_{0}$ denotes local dissipation due to inelastic deformation in the rod's cross-section. The quantity $\mathfrak{D}^{\text {cond }}$ can be shown to be nonnegative on applying Fourier's law of conduction. Moreover, $\mathfrak{D}^{\text {loc }}$ is assumed to be non-negative, and is also known as the local dissipation inequality. 


\subsection{Evolution equation for the representative temperature field}

To obtain the evolution equation for the representative temperature of the rod's cross-section, we begin with the constitutive relation for entropy and take its time derivative:

$$
\begin{aligned}
\eta & =-\frac{\partial \hat{\Psi}}{\partial T}\left(\mathbf{v}_{0}, \mathbf{k}_{0}, T, \boldsymbol{\beta}_{0}\right) \\
\Rightarrow \dot{\eta} & =-\frac{\partial^{2} \Psi}{\partial T \partial T} \dot{T}-\frac{\partial^{2} \Psi}{\partial \mathbf{v}_{0} \partial T} \cdot \boldsymbol{\varepsilon}_{0}-\frac{\partial^{2} \Psi}{\partial \mathbf{k}_{0} \partial T} \cdot \varkappa_{0}-\frac{\partial^{2} \Psi}{\partial \boldsymbol{\beta}_{0} \partial T} \cdot \dot{\boldsymbol{\beta}}_{0} \\
\Rightarrow \dot{\eta} & =\frac{1}{T} C_{v} \dot{T}-\partial_{T} \mathbf{n}_{0} \cdot \boldsymbol{\varepsilon}_{0}-\partial_{T} \mathbf{m}_{0} \cdot \varkappa_{0}+\partial_{T} \mathbf{A}_{0} \cdot \dot{\boldsymbol{\beta}}_{0} \\
\text { or: } T \dot{\eta} & =C_{v} \dot{T}-T\left[\partial_{T} \mathbf{n}_{0} \cdot \boldsymbol{\varepsilon}_{0}+\partial_{T} \mathbf{m}_{0} \cdot \varkappa_{0}-\partial_{T} \mathfrak{D}^{\mathrm{loc}}\right] .
\end{aligned}
$$

Here, $C_{v}=-T\left(\partial^{2} \Psi / \partial T^{2}\right)$ has the unit of energy per unit undeformed length and per unit temperature. It also equals the rate of internal energy (per unit undeformed length of the rod) with respect to the representative temperature $T$, which can be proved as follows:

$$
\begin{aligned}
C_{v} & =-T \frac{\partial^{2} \Psi}{\partial T^{2}}=T \frac{\partial \eta}{\partial T} \\
\Rightarrow C_{v} & =\frac{\partial(\eta T)}{\partial T}-\eta=\frac{\partial(\eta T)}{\partial T}+\frac{\partial \Psi}{\partial T} \\
\Rightarrow C_{v} & =\frac{\partial u}{\partial T}
\end{aligned}
$$

It is thus the analog of the bulk specific heat capacity. On comparing equation (25d) with the entropy balance equation (equation (17)), we obtain

$$
\begin{aligned}
\hat{\gamma}-q^{\prime}+\frac{q T^{\prime}}{T}+\mathfrak{D} & =C_{v} \dot{T}-T\left[\partial_{T} \mathbf{n}_{0} \cdot \boldsymbol{\varepsilon}_{0}+\partial_{T} \mathbf{m}_{0} \cdot \varkappa_{0}-\partial_{T} \mathfrak{D}^{\mathrm{loc}}\right] \\
\Rightarrow C_{v} \dot{T} & =\hat{\gamma}-q^{\prime}+\frac{q T^{\prime}}{T}+\mathfrak{D}+T\left[\partial_{T} \mathbf{n}_{0} \cdot \boldsymbol{\varepsilon}_{0}+\partial_{T} \mathbf{m}_{0} \cdot \varkappa_{0}-\partial_{T} \mathfrak{D}^{\mathrm{loc}}\right] \\
\text { or: } C_{v} \dot{T} & =\hat{\gamma}-q^{\prime}+\mathfrak{D}_{\mathrm{loc}}+T\left[\partial_{T} \mathbf{n}_{0} \cdot \boldsymbol{\varepsilon}_{0}+\partial_{T} \mathbf{m}_{0} \cdot \varkappa_{0}-\partial_{T} \mathfrak{D}^{\mathrm{loc}}\right]
\end{aligned}
$$

In the case of thermoelasticity, $\mathfrak{D}_{\text {loc }}$ vanishes. On further using Fourier's law for heat conduction $\left(q=-\kappa T^{\prime}\right)$, this equation simplifies to

$$
C_{v} \dot{T}=\hat{\gamma}+\kappa T^{\prime \prime}+T\left[\partial_{T} \mathbf{n}_{0} \cdot \boldsymbol{\varepsilon}_{0}+\partial_{T} \mathbf{m}_{0} \cdot \varkappa_{0}\right]
$$

Here, $\kappa$ denotes the coefficient of thermal conductivity. The term $\left(\partial_{T} \mathbf{n}_{0} \cdot \boldsymbol{\varepsilon}_{0}+\partial_{T} \mathbf{m}_{0} \cdot \varkappa_{0}\right)$ is responsible for the thermomechanical coupling. In the general case of inelasticity, as in equation (26), we also have local dissipative or structural heating due to the presence of $\mathfrak{D}^{\text {loc }}$, as well as its derivative $\partial_{T} \mathfrak{D}^{\text {loc }}$. The expression of $\mathfrak{D}^{\text {loc }}$ due to plastic deformation will be obtained later.

\section{Thermoelastoplastic deformation of special Cosserat rods}

Having discussed thermodynamics from a general perspective, we now discuss the rod's kinematics specifically for the thermoelastoplastic deformation.

\section{I. Kinematics}

We begin by postulating that the total material strains of the rod can be additively decomposed into elastic and plastic parts, i.e.,

$$
\mathbf{v}_{0}=\mathbf{v}_{0}^{\mathrm{e}}+\mathbf{v}_{0}^{\mathrm{p}}, \quad \mathbf{k}_{0}=\mathbf{k}_{0}^{\mathrm{e}}+\mathbf{k}_{0}^{\mathrm{p}} .
$$


Here, the superscript "e" implies the elastic part of the total strain, whereas the superscript "p" denotes the plastic part. Their spatial counterparts can simply be obtained through rotational push-forwards. Accordingly, the spatial strains also decompose additively into elastic and plastic parts, i.e.,

$$
\mathbf{v}=\mathbf{v}^{\mathrm{e}}+\mathbf{v}^{\mathrm{p}}, \quad \mathbf{k}=\mathbf{k}^{\mathrm{e}}+\mathbf{k}^{\mathrm{p}} .
$$

The quantities $\left(\mathbf{v}_{0}^{\mathrm{p}}, \mathbf{k}_{0}^{\mathrm{p}}\right)$, together with the hardening variables $\boldsymbol{\mu}_{0} \in \mathbf{R}^{m}$, constitute the internal variable vector $\boldsymbol{\beta}_{0}$, i.e., $\boldsymbol{\beta}_{0}=\left[\begin{array}{lll}\mathbf{v}_{0}^{\mathrm{p}} & \mathbf{k}_{0}^{\mathrm{p}} & \boldsymbol{\mu}_{0}\end{array}\right]^{\mathrm{T}}$. Here, $m$ denotes the number of hardening parameters. The physical meaning of $\left(\mathbf{v}_{0}^{\mathrm{e}}, \mathbf{k}_{0}^{\mathrm{e}}\right)$ is the strain by which the total strain of the rod would have to be reduced locally in order to obtain the local state $\left(\mathbf{v}_{0}^{\mathrm{p}}, \mathbf{k}_{0}^{\mathrm{p}}\right)$ of the rod, which would be locally free of internal force and moment in the rod's crosssection. Comparing with the three-dimensional theory of finite elastoplasticity, it is assumed there that the total deformation gradient tensor decomposes multiplicatively into elastic and plastic parts. ${ }^{6}$ However, the elastic and plastic parts do not turn out to be compatible in general, i.e., the configuration of the body generated through just the plastic part of the deformation gradient won't necessarily be compatible. For the same reason, although the total strain measures $\left(\mathbf{v}_{0}, \mathbf{k}_{0}\right)$ of the rod are compatible, i.e., they generate a compatible configuration $(\mathbf{r}, \mathbf{R})$ of the rod; their elastic and plastic parts need not generate compatible configurations individually.

The additive decomposition of the material strain rates follow directly from the time derivative of equation (28), i.e.,

$$
\begin{aligned}
\dot{\mathbf{v}}_{0} & =\dot{\mathbf{v}}_{0}^{\mathrm{e}}+\dot{\mathbf{v}}_{0}^{\mathrm{p}}, & & \dot{\mathbf{k}}_{0}=\dot{\mathbf{k}}_{0}^{\mathrm{e}}+\dot{\mathbf{k}}_{0}^{\mathrm{p}}, \\
\Rightarrow \boldsymbol{\varepsilon}_{0} & =\varepsilon_{0}^{\mathrm{e}}+\boldsymbol{\varepsilon}_{0}^{\mathrm{p}}, & & \varkappa_{0}=\varkappa_{0}^{\mathrm{e}}+\varkappa_{0}^{\mathrm{p}} .
\end{aligned}
$$

We now show that the spatial strain rates also decompose additively:

$$
\begin{aligned}
\dot{\mathbf{v}}=\dot{\mathbf{R v}_{0}} & =\omega \times \mathbf{v}+\mathbf{R} \dot{\mathbf{v}}_{0}=\omega \times\left[\mathbf{v}^{\mathrm{e}}+\mathbf{v}^{\mathrm{p}}\right]+\mathbf{R}\left[\dot{\mathbf{v}}_{0}^{\mathrm{e}}+\dot{\mathbf{v}}_{0}^{\mathrm{p}}\right] \\
& =\left[\omega \times \mathbf{v}^{\mathrm{e}}+\mathbf{R} \dot{\mathbf{v}}_{0}^{\mathrm{e}}\right]+\left[\omega \times \mathbf{v}^{\mathrm{p}}+\mathbf{R} \dot{\mathbf{v}}_{0}^{\mathrm{p}}\right] \\
& =\dot{\mathbf{R v}_{0}^{\mathrm{e}}}+\frac{\dot{\mathbf{R} \mathbf{v}_{0}^{\mathrm{p}}}}{=} \dot{\mathbf{v}}^{\mathrm{e}}+\dot{\mathbf{v}}^{\mathrm{p}} \\
\Rightarrow \varepsilon & =\boldsymbol{\varepsilon}^{\mathrm{e}}+\boldsymbol{\varepsilon}^{\mathrm{p}} .
\end{aligned}
$$

Using exactly the same steps, one can show that $\dot{\mathbf{k}}$ also decomposes additively, i.e.,

$$
\varkappa=\varkappa^{\mathrm{e}}+\varkappa^{\mathrm{p}} \text {. }
$$

\subsection{Thermoelastoplastic constitutive relations}

We now assume the following special form for the Helmholtz energy density $(\Psi)$ :

$$
\begin{aligned}
\hat{\Psi}\left(\mathbf{v}_{0}, \mathbf{k}_{0}, \mathbf{v}_{0}^{\mathrm{p}}, \mathbf{k}_{0}^{\mathrm{p}}, \boldsymbol{\mu}_{0}, T\right) & =\tilde{\Psi}\left(\mathbf{v}_{0}-\mathbf{v}_{0}^{\mathrm{p}}, \mathbf{k}_{0}-\mathbf{k}_{0}^{\mathrm{p}}, T\right)+\frac{1}{2}\left[\mathbf{H}_{0} \boldsymbol{\mu}_{0}\right] \cdot \boldsymbol{\mu}_{0} \\
& =\tilde{\Psi}\left(\mathbf{v}_{0}^{\mathrm{e}}, \mathbf{k}_{0}^{\mathrm{e}}, T\right)+\frac{1}{2}\left[\mathbf{H}_{0} \boldsymbol{\mu}_{0}\right] \cdot \boldsymbol{\mu}_{0} .
\end{aligned}
$$

Here, $\mathbf{H}_{0} \in \mathbf{R}^{m} \times \mathbf{R}^{m}$ denotes the hardening "stiffness tensor" and is assumed to be a constant. The tensor " $\mathbf{H}_{0}$ " is often assumed to be diagonal. We further assume that the remaining part of the energy $\tilde{\Psi}$ only depends on the elastic contribution to the total strain. Thus, $\tilde{\Psi}$ could be a general hyperelastic model for energy. Given equation (33), the local dissipation $\mathfrak{D}^{\text {loc }}$ becomes

$$
\begin{aligned}
\mathfrak{D}^{\text {loc }} & =-\partial_{\boldsymbol{\beta}_{0}} \Psi \cdot \dot{\boldsymbol{\beta}}_{0}=-\partial_{\mathbf{v}_{0}^{\mathrm{p}}} \Psi \cdot \dot{\mathbf{v}}_{0}^{\mathrm{p}}-\partial_{\mathbf{k}_{0}^{\mathrm{p}}} \Psi \cdot \dot{\mathbf{k}}_{0}^{\mathrm{p}}-\partial_{\boldsymbol{\mu}_{0}} \Psi \cdot \dot{\boldsymbol{\mu}}_{0} \\
& =\partial_{\mathbf{v}_{0}} \tilde{\Psi} \cdot \dot{\mathbf{v}}_{0}^{\mathrm{p}}+\partial_{\mathbf{k}_{0}} \tilde{\Psi} \cdot \dot{\mathbf{k}}_{0}^{\mathrm{p}}-\left[\mathbf{H}_{0} \boldsymbol{\mu}_{0}\right] \cdot \dot{\boldsymbol{\mu}}_{0} \\
& =\mathbf{n}_{0} \cdot \boldsymbol{\varepsilon}_{0}^{\mathrm{p}}+\mathbf{m}_{0} \cdot \varkappa_{0}^{\mathrm{p}}+\mathbf{h}_{0} \cdot \dot{\boldsymbol{\mu}}_{0}
\end{aligned}
$$


Here, $\mathbf{h}_{0}:=-\mathbf{H}_{0} \boldsymbol{\mu}_{0}$ denotes the thermodynamic stress resultant conjugate to the hardening variable $\boldsymbol{\mu}_{0}$. Furthermore,

$$
\partial_{T} \mathfrak{D}_{\text {loc }}=\partial_{T} \mathbf{n}_{0} \cdot \boldsymbol{\varepsilon}_{0}^{\mathrm{p}}+\partial_{T} \mathbf{m}_{0} \cdot \varkappa_{0}^{\mathrm{p}} .
$$

Substituting equations (34) and (35) into equation (26c), we finally obtain the following equation for the evolution of the temperature field:

$$
C_{v} \dot{T}=\hat{\gamma}-q^{\prime}+\mathbf{n}_{0} \cdot \boldsymbol{\varepsilon}_{0}^{\mathrm{p}}+\mathbf{m}_{0} \cdot \varkappa_{0}^{\mathrm{p}}+\mathbf{h}_{0} \cdot \dot{\mu}_{0}+T\left[\partial_{T} \mathbf{n}_{0} \cdot \boldsymbol{\varepsilon}_{0}^{\mathrm{e}}+\partial_{T} \mathbf{m}_{0} \cdot \varkappa_{0}^{\mathrm{e}}\right] .
$$

4.2.I. Quadratic constitutive model for the stored energy $\tilde{\Psi}$. We now present the quadratic form of $\tilde{\Psi}$, which is obtained by its Taylor's expansion up to quadratic order:

$$
\begin{aligned}
\tilde{\Psi}=\tilde{\Psi}_{0}+\frac{\partial \tilde{\Psi}}{\partial T}\left[T-T_{0}\right]+\frac{\partial \tilde{\Psi}}{\partial\left[\mathbf{v}_{0}, \mathbf{k}_{0}\right]} \cdot\left[\begin{array}{l}
\mathbf{v}_{0}-\mathbf{v}_{0}^{\mathrm{p}} \\
\mathbf{k}_{0}-\mathbf{k}_{0}^{\mathrm{p}}
\end{array}\right]+\frac{1}{2} \frac{\partial^{2} \tilde{\Psi}}{\partial T^{2}}\left[T-T_{0}\right]^{2} \\
+\frac{\partial^{2} \tilde{\Psi}}{\partial T \partial\left[\mathbf{v}_{0}, \mathbf{k}_{0}\right]} \cdot\left[\begin{array}{l}
\mathbf{v}_{0}-\mathbf{v}_{0}^{\mathrm{p}} \\
\mathbf{k}_{0}-\mathbf{k}_{0}^{\mathrm{p}}
\end{array}\right]\left[T-T_{0}\right]+\frac{1}{2}\left[\frac{\partial^{2} \tilde{\Psi}}{\partial\left[\mathbf{v}_{0}, \mathbf{k}_{0}\right] \partial\left[\mathbf{v}_{0}, \mathbf{k}_{0}\right]}\left[\begin{array}{c}
\mathbf{v}_{0}-\mathbf{v}_{0}^{\mathrm{p}} \\
\mathbf{k}_{0}-\mathbf{k}_{0}^{\mathrm{p}}
\end{array}\right]\right] \cdot\left[\begin{array}{l}
\mathbf{v}_{0}-\mathbf{v}_{0}^{\mathrm{p}} \\
\mathbf{k}_{0}-\mathbf{k}_{0}^{\mathrm{p}}
\end{array}\right]
\end{aligned}
$$

Here, $\tilde{\Psi}_{0}$ denotes the energy density corresponding to $\left(\mathbf{v}_{0}^{\mathrm{e}}=\mathbf{e}_{3}, \mathbf{k}_{0}^{\mathrm{e}}=\mathbf{0}, T=T_{0}\right)$. Furthermore, all the derivatives of $\tilde{\Psi}$ on the right-hand side of this expression are also taken at $\left(\mathbf{v}_{0}^{\mathrm{e}}=\mathbf{e}_{3}, \mathbf{k}_{0}^{\mathrm{e}}=\mathbf{0}, T=T_{0}\right)$. This quadratic form of energy holds in the regime where elastic strains are small enough and the temperature $T$ is close to the reference temperature $T_{0}$. Assuming that there are no residual forces and moments when the elastic part of the total strain is zero implies that $\partial \tilde{\Psi} / \partial\left[\mathbf{v}_{0}, \mathbf{k}_{0}\right]=\mathbf{0}$. The term $\partial^{2} \tilde{\Psi} / \partial T \partial\left[\mathbf{v}_{0}, \mathbf{k}_{0}\right]$ denotes the thermomechanical coupling stiffness, whereas $\partial^{2} \tilde{\Psi} / \partial\left[\mathbf{v}_{0}, \mathbf{k}_{0}\right] \partial\left[\mathbf{v}_{0}, \mathbf{k}_{0}\right]$ denotes the usual elasticity tensor of the theory of special Cosserat rods.

Using the symmetry group relevant to circular rods, Healey [36] classifies such rods as transversely hemitropic and transversely isotropic rods. Here, we extend this idea to the symmetry group of thermoelastoplastic hemitropic rods and define the relevant symmetry group as

$$
\mathcal{G} \equiv\left\{\mathbf{Q}_{\theta}: \tilde{\Psi}\left(\mathbf{Q}_{\theta} \mathbf{v}_{0}^{\mathrm{e}}, \mathbf{Q}_{\theta} \mathbf{k}_{0}^{\mathrm{e}}, T\right)=\tilde{\Psi}\left(\mathbf{v}_{0}^{\mathrm{e}}, \mathbf{k}_{0}^{\mathrm{e}}, T\right), \forall \theta \in[0,2 \pi], \mathbf{Q}_{\theta} \in \operatorname{SO}(3), \mathbf{Q}_{\theta} \mathbf{e}_{3}=\mathbf{e}_{3}\right\}
$$

Here, $\mathbf{Q}_{\theta}$ denotes rotation by an angle of $\theta$ about the $\mathbf{e}_{3}$ axis. The group $\mathcal{G}$ in equation (38) can make a distinction between left- and right-handed helical patterns in a rod and is useful in modeling intrinsically twisted rods, e.g., chiral nanotubes, DNA, or collagen fibrils. Using the symmetry group of hemitropic rods and a certain "flip-symmetry" that models homogeneity in a rod along its arc-length, one can show that several terms in $\partial^{2} \tilde{\Psi} / \partial T \partial\left[\mathbf{v}_{0}, \mathbf{k}_{0}\right]$ and $\partial^{2} \tilde{\Psi} / \partial\left[\mathbf{v}_{0}, \mathbf{k}_{0}\right] \partial\left[\mathbf{v}_{0}, \mathbf{k}_{0}\right]$ vanish (see Healey [36] and Kumar and Mukherjee [17] for the proof of vanishing of the terms in the elasticity tensor). We then obtain the following simplified quadratic form for $\tilde{\Psi}$ :

$$
\begin{aligned}
\tilde{\Psi}(\cdot)=\tilde{\Psi}_{0}- & \eta_{0}\left[T-T_{0}\right]-\frac{1}{2 T_{0}} \mathcal{S}_{0}\left[T-T_{0}\right]^{2}+\mathcal{L}\left[\mathrm{v}_{0,3}^{\mathrm{e}}-1\right]\left[T-T_{0}\right]+\mathcal{M} k_{0,3}^{\mathrm{e}}\left[T-T_{0}\right] \\
+ & \frac{1}{2}\left[\mathcal{A} k_{0, \alpha}^{\mathrm{e}} k_{0, \alpha}^{\mathrm{e}}+\mathcal{B} k_{0,3}^{\mathrm{e}} k_{0,3}^{\mathrm{e}}+\mathcal{C} \mathrm{v}_{0, \alpha}^{\mathrm{e}} \mathrm{v}_{0, \alpha}^{\mathrm{e}}+\mathcal{D}\left[\mathrm{v}_{0,3}^{\mathrm{e}}-1\right]^{2}+2 \mathcal{E}\left[\mathrm{v}_{0,3}^{\mathrm{e}}-1\right] k_{0,3}^{\mathrm{e}}+2 \mathcal{F} \mathrm{v}_{0, \alpha}^{\mathrm{e}} k_{0, \alpha}^{\mathrm{e}}\right]
\end{aligned}
$$

The scalar $\eta_{0}$ in this equation denotes entropy per unit undeformed length, whereas $\mathcal{S}_{0}$ denotes internal energy per unit undeformed length and per unit temperature; both in the reference state corresponding to $\left(\mathbf{v}_{0}^{\mathrm{e}}=\mathbf{e}_{3}, \mathbf{k}_{0}^{\mathrm{e}}=\mathbf{0}, T=T_{0}\right)$. The coefficients $\mathcal{A}, \mathcal{B}, \mathcal{C}$, and $\mathcal{D}$ denote the bending, twisting, shearing, and extensional stiffnesses, respectively. The coefficient $\mathcal{E}$ denotes coupling between axial strain and twist, whereas $\mathcal{F}$ denotes coupling between bending and shear. Similarly, $\mathcal{L}$ and $\mathcal{M}$ denote the thermomechanical coupling constants: $\mathcal{L}$ denotes the usual coupling between axial strain and temperature, whereas $\mathcal{M}$ denotes coupling between twist and temperature. The coupling coefficients $(\mathcal{E}, \mathcal{F}, \mathcal{M})$ vanish in the case of transversely isotropic 
rods whose symmetry group also contains improper rotation in addition to equation (38). With the quadratic form of equation (39), we now have the following expression for $\left(\mathbf{n}_{0}, \mathbf{m}_{0}, \eta\right)$ :

$$
\begin{aligned}
\mathbf{n}_{0} & =\left[\mathcal{C} \mathrm{v}_{0, \alpha}^{\mathrm{e}}+\mathcal{F} k_{0, \alpha}^{\mathrm{e}}\right] \mathbf{e}_{\alpha}+\left[\mathcal{D}\left[\mathrm{v}_{0,3}^{\mathrm{e}}-1\right]+\mathcal{E} k_{0,3}^{\mathrm{e}}+\mathcal{L}\left[T-T_{0}\right]\right] \mathbf{e}_{3}, \\
\mathbf{m}_{0} & =\left[\mathcal{A} k_{0, \alpha}^{\mathrm{e}}+\mathcal{F} \mathrm{v}_{0, \alpha}^{\mathrm{e}}\right] \mathbf{e}_{\alpha}+\left[\mathcal{B} k_{0,3}^{\mathrm{e}}+\mathcal{E}\left[\mathrm{v}_{0,3}^{\mathrm{e}}-1\right]+\mathcal{M}\left[T-T_{0}\right]\right] \mathbf{e}_{3}, \\
\eta & =\eta_{0}+\frac{1}{T_{0}} \mathcal{S}_{0}\left[T-T_{0}\right]-\left[\mathcal{L}\left[\mathrm{v}_{0,3}^{\mathrm{e}}-1\right]+\mathcal{M} k_{0,3}^{\mathrm{e}}\right]
\end{aligned}
$$

\subsection{Yield function, flow rule, and the hardening law}

The yield function, in general, depends on all the stress resultants present in the reduced form of the local dissipation function (equation (34)). We thus assume the yield function to have the following functional dependence:

$$
\Phi=\hat{\Phi}\left(\mathbf{n}_{0}, \mathbf{m}_{0}, \mathbf{h}_{0} ; T\right) .
$$

Assuming the flow rule to be associative, we thus have the following evolution laws for the plastic strain measures and hardening variables:

$$
\begin{aligned}
\varepsilon_{0}^{\mathrm{p}} & =\lambda \partial_{\mathbf{n}_{0}} \hat{\Phi}, \\
\varkappa_{0}^{\mathrm{p}} & =\lambda \partial_{\mathbf{m}_{0}} \hat{\Phi}, \\
\dot{\mu}_{0} & =\lambda \partial_{\mathbf{h}_{0}} \hat{\Phi} .
\end{aligned}
$$

Here, $\lambda$ denotes the plastic multiplier or the consistency parameter, which vanishes when $\Phi<0$; it further satisfies the following Kuhn-Tucker condition:

$$
\lambda \geq 0 ; \quad \Phi \leq 0 ; \quad \lambda \Phi=0 .
$$

The deformations are assumed to be such that the yield function always remains non-positive, i.e., $\Phi \leq 0$, which leads to rate-independent plasticity.

To introduce isotropic and kinematic hardening, we then assume $\mathbf{h}_{0}=\left[h_{0} \mathbf{h}_{0}^{n} \mathbf{h}_{0}^{m}\right]$. Here the scalar $h_{0}$ models isotropic hardening, whereas the vectors $\mathbf{h}_{0}^{n}$ and $\mathbf{h}_{0}^{m}$ model kinematic hardening. The vectors $\mathbf{h}_{0}^{n}$ and $\mathbf{h}_{0}^{m}$ can also be thought of as the negative of the "back stress resultants" corresponding to $\mathbf{n}_{0}$ and $\mathbf{m}_{0}$, respectively. The hardening variable $\boldsymbol{\mu}_{0}$ can accordingly be decomposed as $\boldsymbol{\mu}_{0}=\left[\mu_{0} \boldsymbol{\mu}_{0}^{n} \boldsymbol{\mu}_{0}^{m}\right]$. Finally, the yield function is specialized as follows to include the two forms of hardening:

$$
\hat{\Phi}\left(\mathbf{n}_{0}, \mathbf{m}_{0}, \mathbf{h}_{0} ; T\right)=\varphi\left(\mathbf{n}_{0}+\mathbf{h}_{0}^{n}, \mathbf{m}_{0}+\mathbf{h}_{0}^{m} ; T\right)-\varsigma_{y}\left(h_{0}\right) .
$$

Here, the function $\varsigma_{y}$ denotes the yield strength function, which depends on the isotropic hardening parameter $h_{0}$. With this form for kinematic hardening, it is easy to deduce that the plastic strain rates and the corresponding kinematic hardening rates match, i.e.,

$$
\begin{aligned}
\boldsymbol{\varepsilon}_{0}^{\mathrm{p}} & =\dot{\boldsymbol{\mu}}_{0}^{n}=\lambda \partial_{\mathbf{n}_{0} \varphi,}, \\
\varkappa_{0}^{\mathrm{p}} & =\dot{\boldsymbol{\mu}}_{0}^{m}=\lambda \partial_{\mathbf{m}_{0}} \varphi .
\end{aligned}
$$

Using equation (34), the local dissipation $\mathfrak{D}^{\text {loc }}$ then becomes

$$
\mathfrak{D}^{\text {loc }}=\lambda\left[\left[\mathbf{n}_{0}+\mathbf{h}_{0}^{n}\right] \cdot \partial_{\mathbf{n}_{0}} \varphi+\left[\mathbf{m}_{0}+\mathbf{h}_{0}^{m}\right] \cdot \partial_{\mathbf{m}_{0}} \varphi-h_{0} \zeta_{y}^{\prime}\right] .
$$

If we further assume $\varphi$ to be homogeneous and of degree one in all its arguments and $\varsigma_{y}$ to be non-homogeneous but also of degree one in $h_{0}$, this equation further simplifies to

$$
\mathfrak{D}^{\text {loc }}=\lambda\left[\varphi-\left[h_{0} \varsigma_{y}^{\prime}+\varsigma_{y}(0)\right]+\varsigma_{y}(0)\right]=\lambda\left[\hat{\Phi}+\varsigma_{y}(0)\right]=\lambda \varsigma_{y}(0) .
$$

Thus, $\mathfrak{D}^{\text {loc }}$ becomes proportional to the initial yield strength. In the absence of hardening, we simply have

$$
\hat{\Phi}\left(\mathbf{n}_{0}, \mathbf{m}_{0} ; T\right)=\varphi\left(\mathbf{n}_{0}, \mathbf{m}_{0} ; T\right)-\varsigma_{y},
$$

which corresponds to the case of ideal plasticity. Here, the yield strength function $\varsigma_{y}$ simply becomes constant. 


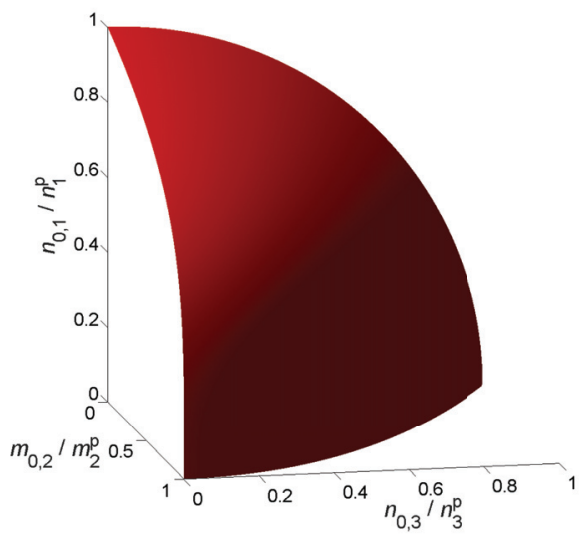

Figure 2. Yield function representation for square cross-section rods in the space of normalized shear force, axial force, and bending moment, as proposed by [34].

4.3.I. Existing forms of yield function. Several authors have proposed approximate yield functions (without hardening) for planar elastoplastic deformations of beams [34, 37-40]. They show that their yield functions match the exact yield surface obtained from the linearized solution of beams subjected to pure bending, torsion, cantilevered loading, etc. We present one such yield function, as proposed by Simo et al. [34], for planar deformation (in the $\mathbf{e}_{1}-\mathbf{e}_{3}$ plane) of the rod having a rectangular cross-section:

$$
\hat{\Phi}\left(n_{0,1}, n_{0,2}=0, n_{0,3}, m_{0,1}=0, m_{0,2}, m_{0,3}=0\right)=\left|\frac{m_{0,2}}{m_{2}^{\mathrm{p}}}\right|+\left[\frac{n_{0,3}}{n_{3}^{\mathrm{p}}}\right]^{2}\left[1+\left[\frac{n_{0,1}}{n_{1}^{\mathrm{p}}}\right]^{2}\right]+\left[\frac{n_{0,1}}{n_{1}^{\mathrm{p}}}\right]^{4}-1
$$

Here, $n_{1}^{\mathrm{p}}$ is the fully plastic shear force, $n_{3}^{\mathrm{p}}$ is the fully plastic axial force, and, finally, $m_{2}^{\mathrm{p}}$ is the fully plastic bending moment. The yield function is represented in Figure 2. Duan and Chen [39] also proposed the following approximate yield function for doubly symmetric cross-sections under biaxial bending and stretching:

$$
\hat{\Phi}\left(n_{0,1}=0, n_{0,2}=0, n_{0,3}, m_{0,1}, m_{0,2}, m_{0,3}=0\right)=\left[\frac{\frac{m_{0,1}}{m_{1}^{\mathrm{p}}}}{1-\left[\frac{n_{0,1}}{n_{1}^{\mathrm{p}}}\right]^{\beta_{1}}}\right]^{\alpha_{1}}+\left[\frac{\frac{m_{0,2}}{m_{2}^{\mathrm{p}}}}{1-\left[\frac{n_{0,1}}{n_{1}^{\mathrm{p}}}\right]^{\beta_{2}}}\right]^{\alpha_{2}}-1 .
$$

Here, $\left(\alpha_{1}, \alpha_{2}, \beta_{1}, \beta_{2}\right)$ are scalar parameters, which depend on the cross-section's shape and are obtained by fitting the yield surface obtained from the linearized solution with the above function. The graphical representation of this yield function is qualitatively very similar to the one in equation (49) (also, see Figure 2). In the case of kinematic hardening, a simple extension would be to replace $\mathbf{n}_{0}$ with $\mathbf{n}_{0}+\mathbf{h}_{0}^{n}$ and $\mathbf{m}_{0}$ with $\mathbf{m}_{0}+\mathbf{h}_{0}^{m}$. The fully plastic forces and moments $\left(n_{1}^{\mathrm{p}}, n_{2}^{\mathrm{p}}, n_{3}^{\mathrm{p}}, m_{1}^{\mathrm{p}}, m_{2}^{\mathrm{p}}, m_{3}^{\mathrm{p}}\right)$ would also evolve in time, which could be categorized under isotropic hardening.

Although the yield functions (equations (49) and (50)) were proposed in an ad-hoc manner and were shown to fit reasonably well with the yield surface obtained numerically from the linearized solution of isotropic beams, deducing an accurate yield function for general three-dimensional deformations and for general material behavior is not a trivial task. Furthermore, at large values of stress resultants $\left(\mathbf{n}_{0}, \mathbf{m}_{0}\right)$, the yield surface obtained from the linearized solution may be inaccurate. Thus, one may have to solve the three-dimensional nonlinear elastoplastic equations in the beam's cross-section.

\section{Conclusions and discussion}

We proposed a general framework for modeling thermoelastoplastic deformations within the special Cosserat rod theory. For the purpose of elastoplastic deformations, it was assumed that the total strain measures $\left(\mathbf{v}_{0}, \mathbf{k}_{0}\right)$ of the special Cosserat rod decompose additively into elastic and plastic parts. We also assumed that the stored energy of the rod depends only on the elastic part of the total strain. We then presented the most general form of the quadratic stored energy for thermoelastoplastic deformations of hemitropic rods. The form of the 
yield function was also discussed, accounting for isotropic and kinematic hardening in the special Cosserat rod framework.

We have presented the theory without establishing its connection to the three-dimensional theory of thermoelastoplasticity. However, such a connection would be necessary in deducing the parameters present in the quadratic stored energy model in equation (39). Kumar et al. [6] obtained these parameters for the purely elastic case by subjecting the rod to uniform strain measures of the rod and further minimizing its cross-sectional energy. Their methodology would need to be generalized to obtain the exact thermoelastic constitutive relations. The connection to the three-dimensional theory would also be necessary in obtaining the mathematical form of the yield function and the hardening law for general three-dimensional deformations of the special Cosserat rod. Finally, the physical meaning of the representative temperature $T$ in equation (18) needs to be made precise. In fact, the definition of internal and external entropy supply rate in equation (16) may be modified, given that the representative temperature $T$ is not the local absolute temperature within the rod's cross-section.

\section{Funding}

This work was supported by the University Grants Commission of India for the Indo-German project (grant number 1-3/2016 (IC)), the DAAD exchange program "Multiscale Modeling, Simulation, and Optimization for Energy, Advanced Materials and Manufacturing," and the Deutsche Forschungsgemeinschaft within the Cluster of Excellence "Engineering of Advanced Materials" (project EXC 315) (Bridge Funding).

\section{ORCID iD}

Ajeet Kumar http://orcid.org/0000-0003-1501-4889

\section{Notes}

1. We refer the readers to Antman [18] for a detailed discussion on this topic.

2. From now on, the rotational pull-back of a vector $\mathbf{a}$ will be denoted by $\mathbf{a}_{0}$ and defined by $\mathbf{a}_{0}=\mathbf{R}^{\mathrm{T}} \mathbf{a}$, whereas that of a secondorder tensor will be defined by $\mathbf{A}_{0}=\mathbf{R}^{\mathrm{T}} \mathbf{A R}$. As a result of this definition, the components of $\mathbf{a}_{0}$ in the global frame $\left(\mathbf{e}_{1}, \mathbf{e}_{2}, \mathbf{e}_{3}\right)$ will be the same as the corresponding components of $\mathbf{a}$ in the local frame of directors $\left(\mathbf{d}_{1}, \mathbf{d}_{2}, \mathbf{d}_{3}\right)$.

3. Note the difference between $\mathbf{v}$ (the strain) and $\boldsymbol{v}$ (the velocity).

4. The proof for the time derivative of the rotational kinetic energy is given in the appendix.

5. Later on, we will introduce plastic strain measures and hardening variables as internal variables.

6. To make this decomposition unique, the plastic part of the deformation gradient is often assumed to be such that it comprises no rotation in its polar decomposition.

\section{References}

[1] Manning, RS, Maddocks, JH, and Kahn, JD. A continuum rod model of sequence-dependent DNA structure. J Chem Phys 1996; 105(13): 5626-5646.

[2] Swigon, D, Coleman, BD, and Tobias, I. The elastic rod model for DNA and its application to the tertiary structure of DNA minicircles in mononucleosomes. Biophys $J$ 1998; 74(5): 2515-2530.

[3] Bozec, L, van der Heijden, G, and Horton, M. Collagen fibrils: Nanoscale ropes. Biophys J 2007; 92: 70-75.

[4] Marko, JF, and Neukirch, S. Competition between curls and plectonemes near the buckling transition of stretched supercoiled DNA. Phys Rev E 2012; 85(1): 011908.

[5] Kumar, A, Mukherjee, S, Paci, JT, et al. A rod model for three dimensional deformations of single-walled carbon nanotubes. Int J Sol Struct 2011; 48: 2849-2858.

[6] Kumar, A, Kumar, S, and Gupta, P. A helical Cauchy-Born rule for special Cosserat rod modeling of nano and continuum rods. J Elast 2016; 124(1): 81-106.

[7] Gupta, P, and Kumar, A. Effect of material nonlinearity on spatial buckling of nanorods and nanotubes. J Elast 2017; 126(2): 155-171.

[8] Audoly, B, and Pomeau, Y. Elasticity and geometry: From hair curls to the non-linear response of shells. Oxford: Oxford University Press, 2010.

[9] McMillen, T, and Goriely, A. Tendril perversion in intrinsically curved rods. J Nonlinear Sci 2002; 12(3): $241-281$.

[10] Costello, GA. Theory of wire rope. New York: Springer-Verlag, 1997.

[11] Goyal, S, Perkins, C, and Lee, CL. Nonlinear dynamics and loop formation in Kirchoff rods with implications to the mechanics of DNA and cables. J Comp Phys 2005; 209: 371-389. 
[12] Chang, J, Pan, J, and Zhang, J. Modelling rod-like flexible biological tissues for medical training. In: Magnenat-Thalmann, N (ed.) Modelling the physiological human 3DPH 2009 (Lecture Notes in Computer Science, vol. 5903). Berlin: Springer, 2009, 51-61.

[13] Love, AEH. A treatise on the mathematical theory of elasticity. Mineola: Dover Books, 2000.

[14] Cosserat, E, and Cosserat, F. Theory of deformable bodies. Paris: Scientific Library A. Hermann and Sons, 1909.

[15] Green, AE, Naghdi, PM, and Wenner, ML. On the theory of rods. I. Derivations from the three-dimensional equations. Proc $R$ Soc London, Ser A 1974; 337(1611): 451-483.

[16] Rubin, MB. Cosserat theories: Shells, rods and points. Dordrecht: Springer Science \& Business Media, 2000.

[17] Kumar, A, and Mukherjee, S. A geometrically exact rod model including in-plane cross-sectional deformation. J App Mech 2011; 78: 011010.

[18] Antman, SS. Nonlinear problems of elasticity. New York: Springer-Verlag, 1995.

[19] Ericksen, JL. Hypo-elastic potentials. Q J Mech Appl Math 1958; 11(1): 67-72.

[20] Green, AE. Micro-materials and multipolar continuum mechanics. Int J Eng Sci 1965; 3(5): 533-537.

[21] Hodges, DH. Nonlinear composite beam theory. Prog Astronaut Aeronaut 2006; 213: 304.

[22] Berdichevsky, V. Variational principles of continuum mechanics: I. Fundamentals. Heidelberg: Springer, 2009.

[23] DeSilva, CN, and Whitman, AB. Thermodynamical theory of directed curves. J Math Phys 1971; 12(8): $1603-1609$.

[24] Kafadar, CB. On the nonlinear theory of rods. Int J Eng Sci 1972; 10(4): 369-391.

[25] Green, AE, and Naghdi, PM. On thermal effects in the theory of rods. Int J Solids Struct 1979; 15(11): 829-853.

[26] Simmonds, JG. A simple nonlinear thermodynamic theory of arbitrary elastic beams. J Elast 2005; 81(1): 51-62.

[27] Beyrouthy, J, and Neff, P. A viscoelastic thin rod model for large deformations: Numerical examples. Math Mech Solids 2011; 16(8): 887-896.

[28] Linn, J, Lang, H, and Tuganov, A. Geometrically exact Cosserat rods with Kelvin-Voigt type viscous damping. Mech Sci 2013; 4(1): 79-96.

[29] Altenbach, H, Bîrsan, M, and Eremeyev, VA. On a thermodynamic theory of rods with two temperature fields. Acta Mech 2012; 223(8): 1583-1596.

[30] Cao, DQ, Song, MT, Tucker, RW, et al. Dynamic equations of thermoelastic Cosserat rods. Commun Nonlinear Sci Numer Simul 2013; 18(7): 1880-1887.

[31] Simo, JC, and Kennedy, JG. On a stress resultant geometrically exact shell model. Part V. Nonlinear plasticity: Formulation and integration algorithms. Comp Methods App Mech Eng 1992; 96(2): 133-171.

[32] Saje, M, Planinc, I, Turk, G, et al. A kinematically exact finite element formulation of planar elastic-plastic frames. Comp Methods App Mech Eng 1997; 144 (1-2): 125-151.

[33] Štok, B, and Halilovi, M. Analytical solutions in elasto-plastic bending of beams with rectangular cross section. Appl Math Modell 2009; 33(3): 1749-1760.

[34] Simo, JC, Hjelmstad, KD, and Taylor, RL. Numerical formulations of elasto-viscoplastic response of beams accounting for the effect of shear. Comp Methods App Mech Eng 1984; 42(3): 301-330.

[35] Park, MS, and Lee, BC. Geometrically non-linear and elastoplastic three-dimensional shear flexible beam element of von-Misestype hardening material. Int J Numer Methods Eng 1996; 39(3): 383-408.

[36] Healey, TJ. Material symmetry and chirality in nonlinearly elastic rods. Math Mech Solids 2002; 7: 405-420.

[37] Drucker, DC. The effect of shear on the plastic bending of beams. J Appl Mech 1956; 28: 509.

[38] Neal, BG. The effect of shear and normal forces on the fully plastic moment of a beam of rectangular cross section. J Appl Mech 1961; 28(2): 269-274.

[39] Duan, L, and Chen, W. A yield surface for doubly symmetrical sections. Eng Struct 1990; 12(2): 114-119.

[40] Gendy, AS and Saleeb, AF. Generalized yield surface representations in the elasto-plastic three-dimensional analysis of frames. Comput Struct 1993; 49(2): 351-362.

\section{Appendix: Time derivative of rotational kinetic energy and spatial derivative of angular velocity}

The time derivative of the rotational kinetic energy can be written as

$$
\begin{aligned}
\frac{1}{2} \overline{[\mathbf{I} \boldsymbol{\omega}] \cdot \boldsymbol{\omega}} & =\frac{1}{2}[\dot{[\mathbf{I} \boldsymbol{\omega}]} \cdot \boldsymbol{\omega}+[\mathbf{I} \boldsymbol{\omega}] \cdot \dot{\boldsymbol{\omega}}] \\
& =\frac{1}{2}[\dot{[\mathbf{I} \boldsymbol{\omega}]} \cdot \omega+[\mathbf{I} \dot{\boldsymbol{\omega}}] \cdot \boldsymbol{\omega}]
\end{aligned}
$$


Furthermore,

$$
\begin{aligned}
\mathbf{I} \dot{\boldsymbol{\omega}} & =\dot{\overline{\mathbf{I}} \boldsymbol{\omega}}-\dot{\mathbf{I} \omega} \\
& =\dot{\overline{\mathbf{I}} \boldsymbol{\omega}}-\overline{\mathbf{R I}_{0} \mathbf{R}^{\mathrm{T}} \boldsymbol{\omega}} \\
& =\dot{\mathbf{I} \boldsymbol{\omega}}-\dot{\mathbf{R}} \mathbf{I}_{0} \mathbf{R}^{\mathrm{T}} \boldsymbol{\omega}-\mathbf{R} \mathbf{I}_{0} \dot{\mathbf{R}}^{\mathrm{T}} \boldsymbol{} \\
& =\dot{\mathbf{I} \boldsymbol{\omega}}-\dot{\mathbf{R}} \mathbf{R}^{\mathrm{T}} \mathbf{R} \mathbf{I}_{0} \mathbf{R}^{\mathrm{T}} \boldsymbol{\omega}-\mathbf{R} \mathbf{I}_{0} \mathbf{R}^{\mathrm{T}} \mathbf{R} \dot{\mathbf{R}}^{\mathrm{T}} \boldsymbol{\omega} \\
& =\dot{\overline{\mathbf{I}} \boldsymbol{\omega}}-\boldsymbol{\omega} \times[\mathbf{I} \boldsymbol{\omega}]-\mathbf{R} \mathbf{I}_{0} \mathbf{R}^{\mathrm{T}}[-\boldsymbol{\omega} \times \boldsymbol{\omega}] \\
& =\dot{\mathbf{I} \boldsymbol{\omega}}-\boldsymbol{\omega} \times[\mathbf{I} \boldsymbol{\omega}] .
\end{aligned}
$$

Substituting equation (52) into equation (51), we then obtain

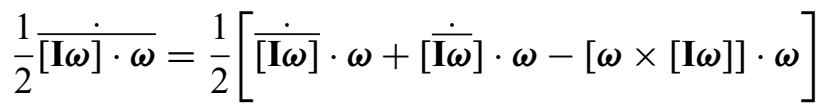

$$
\begin{aligned}
& =[\dot{[\mathbf{I} \omega]} \cdot \omega] \text {. }
\end{aligned}
$$

Similarly, to show that $\boldsymbol{\omega}^{\prime}=\mathbf{R} \dot{\mathbf{k}}_{0}$, we begin with the spatial derivative of the skew-symmetric tensor $\mathbf{W}=\dot{\mathbf{R}} \mathbf{R}^{\mathrm{T}}$ :

$$
\begin{aligned}
& \mathbf{W}^{\prime}=\left[\dot{\mathbf{R}} \mathbf{R}^{\mathrm{T}}\right]^{\prime} \\
& =[\dot{\mathbf{R}}]^{\prime} \mathbf{R}^{\mathrm{T}}+\dot{\mathbf{R}}\left[\mathbf{R}^{\mathrm{T}}\right]^{\prime} \\
& =\dot{\overline{\mathbf{R}^{\prime}}} \mathbf{R}^{\mathrm{T}}+\dot{\mathbf{R}}\left[\mathbf{R}^{\prime}\right]^{\mathrm{T}} \quad \text { (changing the order of derivatives) } \\
& =\overline{\mathbf{R R}^{\mathrm{T}} \mathbf{R}^{\prime}} \mathbf{R}^{\mathrm{T}}+\dot{\mathbf{R}}\left[\mathbf{R} \mathbf{R}^{\mathrm{T}} \mathbf{R}^{\prime}\right]^{\mathrm{T}} \\
& =\dot{\mathbf{R} \mathbf{K}_{0}} \mathbf{R}^{\mathrm{T}}+\dot{\mathbf{R}}\left[\mathbf{R} \mathbf{K}_{0}\right]^{\mathrm{T}} \\
& =\dot{\mathbf{R}} \mathbf{K}_{0} \mathbf{R}^{\mathrm{T}}+\mathbf{R} \dot{\mathbf{K}}_{0} \mathbf{R}^{\mathrm{T}}-\dot{\mathbf{R}} \mathbf{K}_{0} \mathbf{R}^{\mathrm{T}} \\
& \Rightarrow \mathbf{W}^{\prime}=\mathbf{R} \dot{\mathbf{K}}_{0} \mathbf{R}^{\mathrm{T}} \\
& \Rightarrow \omega^{\prime}=\operatorname{axial}\left(\mathbf{W}^{\prime}\right)=\mathbf{R} \dot{\mathbf{k}}_{0} \text {. }
\end{aligned}
$$

